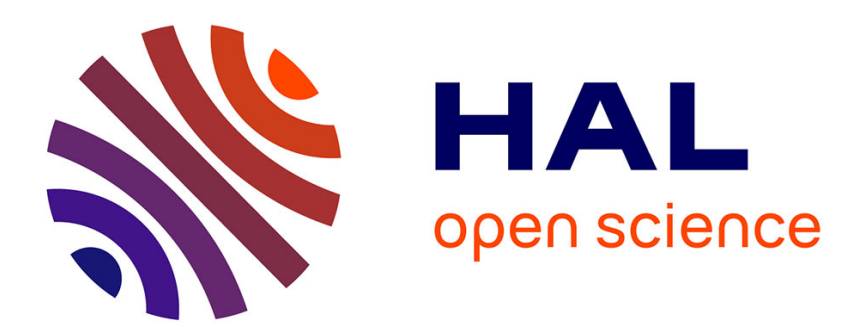

\title{
Control of a Planar Underactuated Biped on a Complete Walking Cycle
}

\author{
Ahmed Chemori, A. Loria
}

\section{To cite this version:}

Ahmed Chemori, A. Loria. Control of a Planar Underactuated Biped on a Complete Walking Cycle. IEEE Transactions on Automatic Control, 2004, 49 (5), pp.838-843. 10.1109/TAC.2004.828314 . lirmm-02360749

\section{HAL Id: lirmm-02360749 https://hal-lirmm.ccsd.cnrs.fr/lirmm-02360749}

Submitted on 13 Nov 2019

HAL is a multi-disciplinary open access archive for the deposit and dissemination of scientific research documents, whether they are published or not. The documents may come from teaching and research institutions in France or abroad, or from public or private research centers.
L'archive ouverte pluridisciplinaire HAL, est destinée au dépôt et à la diffusion de documents scientifiques de niveau recherche, publiés ou non, émanant des établissements d'enseignement et de recherche français ou étrangers, des laboratoires publics ou privés. 


\section{Control of a planar under-actuated biped on a complete walking cycle}

\author{
Ahmed Chemori ${ }^{1} \quad$ Antonio Loría ${ }^{2}$
}

\begin{abstract}
We address the problem of stabilizing a planar biped robot on a complete walking cycle. Our approach is based on singling out the three fundamental phases of motion of a biped : single and double-support, separated (sequentially) by an impact "instantaneous" phase. We propose control laws to drive the robot for a finite time during each phase, while ensuring certain robustness vis-a-vis the impacts which are treated as external perturbations. We also provide some simulation results.
\end{abstract}

\section{INTRODUCTION}

Biped robots have gained an increasing interest in the last few years for many different reasons: they may assist Man in difficult or dangerous tasks in which other mobile robots would fail (e.g. irregular surfaces with multiple obstacles). See the still actual overview 1. From a control viewpoint the problem of making a biped have a dynamically stable walk (i.e., to follow a reference trajectory or path) is interesting due to the complexity of the model: it consists on a set of constrained differential equations and a discrete-time map which induces discontinuity in the solutions. Furthermore, the structure of the system changes depending on the phase of motion 'loosing' or 'gaining' degrees of freedom. Many approaches have been proposed in the literature to address the problem of stable dynamic walking.

Some interesting results smartly exploit the physical properties of the biped. In particular, these results are studied to produce ballistic movements, i.e., the robot with one foot on the ground and one leg swinging in the air is roughly regarded as an inverted pendulum with an equivalent inertia. Then, the control is designed so as to balance the robot forward and to "let it fall" on its swinging foot. Trajectories cannot be planned ahead of time but for an adequate tunning this approach produces periodic movements which are little energy-consuming. See for instance [2, 3, 4]. Other approaches are based on the definition, ahead of time, of the reference trajectories. For instance, in [5] the authors generate the trajectories via a van der Pol oscillator.

The authors are not aware of many rigorous stability proofs for complex biped robots with a model including the equations of impact. A noteworthy example is 6 where the problem is rigorously analyzed via a Poincaré map. See also [7. In particular, the authors propose a criterion for periodic systems with impacts. Another result using a Poincaré criterion is in 8 where the authors propose a sliding mode approach to make the biped follow a nominal trajectory with an asymptotically convergent behavior to a cyclic motion. See also 9 for a study from a hybrid systems viewpoint.

In this paper we address the problem of dynamic stability of a biped for which reference trajectories are defined ahead of time. For clarity of exposition we focus on the case study of a 7-degreesof-freedom planar biped prototype described in 10 however, the results presented may apply to other underactuated mechanical systems. Our control approach borrows from previous results for manipulators with holonomic constraints (cf. [11]) and par-

\footnotetext{
${ }^{1}$ INPG, Laboratoire d'Automatique de Grenoble, BP46 ENSIEG, 38402 St. Martin d'Hères, France

${ }^{2}$ C.N.R.S, Laboratoire de Signaux et Systèmes, Supélec, 91192 Gif sur Yvette, France.
}

tial feedback linearization (cf. [12]) since we address the control problem by proposing a controller for each phase of motion: singlesupport phase (one foot grounded) and double-support phase (both feet grounded). The impacts occurring when the swing leg touches the ground are regarded as "external" perturbations.

The rest of the paper is organized as follows. In next section we present in some detail, the dynamic model of the prototype biped robot. In Section 3 we present our main result. We provide some simulation results in Section 4 and conclude with some remarks in Section 5

\section{DYNAMIC MODEL}

It is generally accepted that a walking cycle can be divided into three sequential phases of motion : single-support (SS) i.e., with one foot grounded; impact, and double-support (DS) i.e., both feet grounded. Accordingly, the dynamic model is composed of three sets of equations, each corresponding to a phase of motion. The models for the single and double-support phases are reduced order Lagrangian models. These are obtained by writing first the model for the seven degrees of freedom system (that is, the robot with both feet "in the air") and then, considering the holonomic constraints (generated by the respective situations of one or both feet grounded) to obtain reduced order models. The Lagrange equations can be written as 13,14 .

$$
H(q) \ddot{q}+C(q, \dot{q}) \dot{q}+G(q)=S u
$$

where $q \in \mathbb{R}^{n}$ is the vector of generalized coordinates, $H(q)$ is the inertia matrix, $C(q, \dot{q})$ contains the centrifugal and Coriolis forces terms, $G(q)$ is the vector of gravitational forces, $u \in \mathbb{R}^{p}$ are the control inputs, $S$ is a "torque-distribution matrix" which is constant, full rank and of unitary norm.

In the case-study of this note, we have 7 degrees of freedom in the phase of flight (both feet in the air during running gaits), 5 degrees of freedom in the SS phase and 3 degrees of freedom in the DS phase. The robot has only 4 actuators (at knees and hip) hence, the system is over-actuated during the DS phase and under-actuated in the other phases.

The constraints generated by one or both feet being grounded are holonomic and restrict the biped's motion to a smooth $(n-m)-$ dimensional submanifold and are defined by the equation

$$
\phi(q)=0
$$

where the function $\phi: \mathbb{R}^{n} \rightarrow \mathbb{R}^{m}$ is at least twice continuously differentiable, and $m$ is the number of holonomic constraints ( 2 or 4 depending on the phase of motion).

Generally speaking the constraints of a mechanical system in contact with a surface and under the action of gravity are expressed as $\phi(q) \geq 0$ and are called unilateral (cf e.g. [15]). The case of $\phi(q)>0$ corresponding to configurations in which the system is separated from the surface (i.e., unconstrained) and the case of $\phi(q)=0$ corresponding as already mentioned, to the case when the system is in contact. They are called unilateral because it is assumed that the resulting forces act in only one sense in the direction normal to the surface (in other words, it is assumed that the body (e.g. the foot) may take off but does not penetrate the surface).

In what follows, we derive reduced-order models for the SS phase ( 2 constraints for planar robots) and DS phase (4 constraints for planar robots) based on the constraints equation (2). To that end, 
following [16] we assume that ${ }^{3}$

(Assumption 1) there exist an operating region $\Omega \subset \mathbb{R}^{n}$ defined as $\Omega:=\Omega_{n c} \times \Omega_{c}$, where $\Omega_{n c}$ is a convex subset of $\mathbb{R}^{n-m}, \Omega_{c}$ is an open subset of $\mathbb{R}^{m}$. We also assume the existence of a function $\psi: \Omega_{n c} \rightarrow \mathbb{R}^{m}$ twice continuously differentiable, such that $\phi\left(q_{n c}, \psi\left(q_{n c}\right)\right)=0$ for all $q_{n c} \in \Omega_{n c}$. Under these conditions, the vector of constrained generalized positions, $q_{c}$, can be uniquely defined by the vector $q_{n c}$ such that $q_{c}=\psi\left(q_{n c}\right)$ for all $q_{n c} \in \Omega_{n c}$.

Under this assumption the matrix $J(q)=\partial \phi(q) / \partial q$ can be partitioned as $J(q)=\left[J_{1}(q), J_{2}(q)\right]$, where $J_{1}(q):=\partial \phi(q) / \partial q_{n c}$, $J_{2}(q):=\partial \phi(q) / \partial q_{c}$ and the Jacobian matrix $J_{2}(q) \in \mathbb{R}^{m \times m}$ never degenerates in the set $\Omega$. Necessary and sufficient conditions for global solubility have been given in [17. based on the implicit function theorems of [18. Thus, without loss of generality we can assume that for all $q \in \Omega$ there exist positive constants $\beta_{1}, \beta_{2}, \beta_{3}$ such that, for all $i=1,2, \ldots, n$,

$$
0<\beta_{1} \leq\left\|J_{2}(q)\right\| \leq\|J(q)\| \leq \beta_{2}, \quad\left\|\frac{\partial J(q)}{\partial q_{i}}\right\|<\beta_{3} .
$$

A reduced-order model is obtained by taking the above constraints into consideration. Following [16] (see also 20]) one obtains

$$
\left\{\begin{array}{l}
H_{*}(q) \ddot{q}_{n c}+C_{*}(q, \dot{q}) \dot{q}_{n c}+G_{*}(q)=M^{\top}(q) S u \\
\lambda=Z(q)\left[C_{\lambda}(q, \dot{q}) \dot{q}_{n c}+G(q)-S u\right]
\end{array}\right.
$$

where the index $*$ expresses the model reduction, $\lambda$ corresponds to the Lagrange multipliers related to the force of constraint, $H_{*}(q):=M^{\top}(q) H(q) M(q), \quad G_{*}(q):=M^{\top}(q) g(q), \quad Z(q):=$ $\left(J(q) H^{-1}(q) J^{\top}(q)\right)^{-1} J(q) H^{-1}(q)$ and

$$
\begin{aligned}
M(q) & :=\left(\begin{array}{c}
I_{n-m} \\
-J_{2}^{-1}(q) J_{1}(q)
\end{array}\right) \\
C_{*}(q, \dot{q}) & :=M^{\top}(q)[H(q) \dot{M}(q)+C(q, \dot{q}) M(q)]
\end{aligned}
$$

hence, $M(q)$ is full column rank if and only if $J_{2}^{-1}(q)$ exists. In such case and only then, the reduced order model is valid.

Interestingly enough, the reduced order model inherits the usual properties of robot manipulator models with only rotational joints: the matrix $H_{*}(q)$ is symmetric positive definite for all $q \in \Omega$, $\dot{H}_{*}(q)-2 C_{*}(q, \dot{q})$ is skew-symmetric for all $(q, \dot{q}) \in \Omega^{2}$ and under (3) we also have the existence of constants $d_{m}, d_{M}, k_{c}>0$ such that $d_{m} I \leq H_{*}(q) \leq d_{M} I$ and $\left\|C_{*}(q, \dot{q})\right\| \leq k_{c}\|\dot{q}\|$.

This model can be used for both phases of single and double support the only difference being the number of constraints and therefore of degrees of freedom. We present below the explicit models corresponding to the single-support and double-support phases respectively. The control laws will be independently designed for each model (resp. phase).

In what follows, we restrict our analysis to the case-study of the planar 7-degrees-of-freedom biped fully described in $[10$, 21] (see also http://www-lag.ensieg.inpg.fr/PRC-Bipedes/) hence, $q:=\left[\begin{array}{lllll}q_{31} & q_{41} & q_{32} & q_{42} & q_{1} x y\end{array}\right]^{\top}$ where $q_{4 i}$ are the coordinates of the shins relative to the thighs, the latter are expressed with respect to the vertical and given by $q_{3 i}, q_{1}$ is the coordinate of the torso with respect to the vertical and $x, y$ are the Cartesian coordinates of the hips on the plane (longitude and height). The

\footnotetext{
${ }^{3}$ This is a general assumption fundamental in problems of constrained mechanical systems and it is verified for the case study considered here.

${ }^{4}$ Notice that the inequality $\left\|J_{2}(q)\right\| \leq\|J(q)\|$ in $(\underline{3)}$ is not obvious. Interested readers are referred to [19], ch. X, sec. 6,7 .
}

coordinates of the stance leg correspond to $q_{31}$ and $q_{41}$. Other constraints not explicitly mentioned here are those of non-sliding. These induce reaction forces tangential to the contact surface, i.e., the ground and are also holonomic

Standing assumption 1 We assume that the feet in contact with the surface do not slide nor leave the surface at "unwanted" moments. In particular, when an impact of the swing foot with the ground occurs, the foot neither slides nor bounces. Also, the support foot does not slide and the stance leg lifts off without interaction.

Dynamics during the single-support phase: In this phase the constraints are expressed by

$$
\left\{\begin{array}{l}
x_{p}=x-l_{3} \sin \left(q_{31}\right)-l_{4} \sin \left(q_{31}+q_{41}\right)=(i-1) L \\
y_{p}=y+l_{3} \cos \left(q_{31}\right)+l_{4} \cos \left(q_{31}+q_{41}\right)=0
\end{array}\right.
$$

where $\left(x_{p}, y_{p}\right)$ denote the coordinates of the support foot, $i$ indexes the step, $L$ denotes the length of the step, $l_{1}$ denotes the length of the torso, $l_{3}$ the length of the thighs and $l_{4}$ the length of the shins. A direct calculation (see 21]) shows that in this case $J_{2}(q) \equiv I$ that is, $\Omega_{n c}=\mathbb{R}^{5} \times \mathbb{R}^{5}$. We stress however, that $u \in \mathbb{R}^{4}$ hence in single-support the system is under-actuated. In the sequel we will refer to $\Omega_{n c}$ for this phase as $\Omega_{S S}$.

Dynamics during the double-support phase: During the double-support phase the biped has both feet grounded, this is expressed by the following 4 constraint equations,

$$
\left\{\begin{array}{l}
x_{p 1}=x-l_{3} \sin \left(q_{31}\right)-l_{4} \sin \left(q_{31}+q_{41}\right)=(i-1) L \\
y_{p 1}=y+l_{3} \cos \left(q_{31}\right)+l_{4} \cos \left(q_{31}+q_{41}\right)=0 \\
x_{p 2}=x-l_{3} \sin \left(q_{32}\right)-l_{4} \sin \left(q_{32}+q_{42}\right)=i L \\
y_{p 2}=y+l_{3} \cos \left(q_{32}\right)+l_{4} \cos \left(q_{32}+q_{42}\right)=0 .
\end{array}\right.
$$

To derive the dynamic model one proceeds as for the model for the SS phase. Hence, the resulting reduced order model has the form (4) but this time $q_{n c} \in \mathbb{R}^{3}$, and it is valid on the space $\Omega_{n c}$ which corresponds to the set where the constraints Jacobian matrix $J_{2}(q)$ is full rank. A simple computation shows that this corresponds to the condition $q_{42} \neq k \pi, k \in \mathbb{N}$, i.e., $\Omega_{n c}=\left\{\left(q_{n c}, \dot{q}_{n c}\right) \in \mathbb{R}^{3} \times \mathbb{R}^{3}: q_{42}\left(q_{n c}\right) \neq k \pi\right\}$. Notice that in this phase the system is over-actuated since it possesses only 3 degrees of freedom. For further analysis, we will denote $\Omega_{n c}$ for this phase by $\Omega_{D S}$.

Impacts model: The impact between the swing foot and the ground is considered as a rigid collision according to [22. One may define the impact condition as that implying that the robot enters in the DS configuration. This is given by equating the second and fourth equations in (7). To obtain the dynamic model for this phase we need irregular mechanics laws (cf. for instance 23]). The contact forces are expressed as impulse forces acting on the Lagrangian dynamics that is,

$$
H(q) \ddot{q}+C(q, \dot{q}) \dot{q}+G(q)=S u+\delta F_{\text {ext }}
$$

where $\delta F_{\text {ext }}$ represents the external contact forces determined by the constraints. Under Standing assumption 1 and the hypotheses that: i) the impact is instantaneous, ii) its effective force can be expressed by an impulse and iii) it leads only to changes in the velocities but the positions remain continuous; one can deduce the following formula of the external forces by integration of (8) over the impact duration i.e.,

$$
H(q)\left(\dot{q}^{+}-\dot{q}^{-}\right)=F_{e x t}(t, q, \dot{q})
$$


where $\dot{q}^{+}$(resp. $\dot{q}^{-}$) denotes the velocity just after (resp. before) an impact. See 22$]$ in the context of planar robots with rotational joints and 23] for a thorough study of impact mechanics. We also have that the forces of constraint do not produce any work hence, denoting the last two equations in $(7)$ by $\phi(q)=0$ and $J(q):=\frac{\partial \phi}{\partial q}$ we have that

$$
J(q) \dot{q}^{+}=0
$$

and $F_{\text {ext }}=J(q)^{\top} \lambda$ where $\lambda:=\operatorname{col}\left[\lambda_{t}, \lambda_{n}\right]$ and $\lambda_{t}, \lambda_{n}$ correspond to the tangential and normal forces at the moment of impact. Solving equation (9) and (10) for $\dot{q}^{+}, \lambda_{t}$, and $\lambda_{n}$ yields an expression for $\dot{q}^{+}$in terms of $\dot{q}^{-}$.

$$
\left[\begin{array}{c}
q^{+} \\
\dot{q}^{+}
\end{array}\right]=\Delta(q)\left[\begin{array}{c}
q^{-} \\
\dot{q}^{-}
\end{array}\right]
$$

where $\Delta(q)=$ block-diag $\left\{W_{1}, W_{2}(q)\right\}$, with $W_{1} \quad:=$ block-diag $\left\{\left[0 I_{2 \times 2} ; I_{2 \times 2} 0\right] I_{3 \times 3}\right\} \quad$ and $\quad W_{2}(q) \quad:=I_{7 \times 7} \quad$ $H^{-1}(q) J^{\top}(q)\left(J(q) H^{-1}(q) J^{\top}(q)\right)^{-1} J(q)$. The choice of $W_{1}$ reflects in particular, that the coordinates between the support and swing legs commute during the impact. Notice that under Assumption $10(q)$ is uniformly bounded; hence, the impact dynamics (11) is globally Lipschitz.

\section{The CONTROL APPROACH}

In few words, the control strategy is as follows: during the SS phase we impose 4 desired reference trajectories for the four limbs and control the latter, leaving the torso to describe a "free" movement. During the DS phase we chose to control one leg and to correct the torso deviations from the vertical. We consider that the impact induces an instantaneous force which may be seen as a state-dependent perturbation. Hence, the control law for the SS phase is designed with a certain degree of robustness in order to ensure that the DS phase starts with appropriate initial conditions in spite of the impact.

\subsection{SS phase control}

To reproduce a human gait we impose a trajectory of inverted pendulum to the support leg while the swing leg follows an "opposite" movement. To generate a periodic motion we choose the initial conditions of each phase as the final conditions of the previous one. Finally, the reference trajectories must obviously lay in the sets of validity for each model, $\Omega_{S S}$ and $\Omega_{D S}$. Under these considerations we propose the following reference trajectories $\left(t_{S S}\right.$ is the duration of the SS phase),

$$
\left\{\begin{array}{l}
q_{31 d}(t)=\frac{1}{2}\left(1-\cos \left(\frac{\pi t}{t_{s s}}\right)\right)\left(q_{32 d}(0)-q_{31 d}(0)\right)+q_{31 d}(0) \\
q_{41 d}(t) \equiv q_{41 d}(0) \\
q_{32 d}(t)=\frac{1}{2}\left(1-\cos \left(\frac{\pi t}{t_{s s}}\right)\right)\left(q_{31 d}(0)-q_{32 d}(0)\right)+q_{32 d}(0) \\
q_{42 d}(t)=\frac{1}{2}\left(1-\cos \left(\frac{2 \pi t}{t_{s s}}\right)\right) \alpha+q_{42 d}(0)
\end{array}\right.
$$

The torso reference trajectory is left to be defined later in a way that it satisfies the closed loop differential equations. The parameter $\alpha$ is used as a tunning variable to generate trajectories which are tested (in simulations) to be compatible with non-sliding and non-bouncing constraints, neglected in the analysis, and to produce a periodic gait. The length of the step is determined by the choice of the initial conditions $q_{d}(0)$. One could also choose to make these values vary at each step in order to allow the length of steps vary with time however, this shall not be pursued here. Observe also that the length of the step determines the average walking velocity for each choice of step duration.

The control strategy is based on one hand, on previous results on control of robots with holonomic constraints -specifically those contained in [11] and on the other, on the technique of partial feedback linearization of $[12]$. Define

$$
u=S^{+}\left(M^{+}\right)^{\top}(q) u_{a}
$$

where $(\cdot)^{+}$stands for the pseudo-inverse of $(\cdot)$. This control law achieves a decoupling of the dependent and the independent dynamics from (4). Regarding only the first set of equations in (4) we have that

$$
H_{*}(q) \ddot{q}_{n c}+C_{*}(q, \dot{q}) \dot{q}_{n c}+G_{*}(q)=u_{a}
$$

where $u_{a}$ is the control inputs for the 5 independent limbs (indexed as $\left.(\cdot)_{n c}\right)$ of the biped however, we remind that only 4 controls are available hence $u_{a}:=\left[u_{1} 0\right]^{\top}, u_{1} \in \mathbb{R}^{4}$. Furthermore, let us partition the independent coordinates in single-support phase into actuated and non actuated variables, i.e., $q_{n c}:=\left[q_{a} q_{n a}\right]^{\top}$. Correspondingly, let $H_{*}:=\left\{h_{11}, h_{12} ; h_{21}, h_{22}\right\}, C_{*}:=\left\{c_{11}, c_{12} ; c_{21}, c_{22}\right\}$, $G_{*}:=\left\{g_{1} ; g_{2}\right\}$. Then, the system dynamics in this phase becomes

$$
\begin{gathered}
h_{11} \ddot{q}_{a}+h_{12} \ddot{q}_{n a}+c_{11} \dot{q}_{a}+c_{12} \dot{q}_{n a}+g_{1}(q)=u_{1} \\
h_{21} \ddot{q}_{a}+h_{22} \ddot{q}_{n a}+c_{21} \dot{q}_{a}+c_{22} \dot{q}_{n a}+g_{2}(q)=0
\end{gathered}
$$

and we stress at this point that there exists $h_{M}>0$ such that for all $q \in \Omega$ we have that $\left\|h_{i j}(q)\right\| \leq h_{M}$ for all $i, j=1,2$. Let $K_{p}$, $K_{d}, K_{p}^{\prime}, K_{d}^{\prime}$ be diagonal positive definite matrices. The partial feedback linearizing control law for the independent coordinates $q_{n c}$ in this phase is given by

$u_{1}=R \ddot{q}_{a d}-h_{12} h_{22}^{-1}\left(g_{2}+c_{21} \dot{q}_{a}+c_{22} \dot{q}_{n a}\right)+g_{1}(q)+c_{11} \dot{q}_{a}+c_{12} \dot{q}_{n a}+u_{2}$

where $R=h_{11}-h_{12} h_{22}^{-1} h_{21}$ is invertible ${ }^{5}$ and $u_{2}=-R\left(K_{d} \dot{\tilde{q}}_{a}+\right.$ $\left.K_{p} \tilde{q}_{a}\right)$ and $(\tilde{\cdot}):=(\cdot)-(\cdot)_{d}$. On the other hand, for the torso we will analyze its stability with respect to the trajectory $q_{\text {nad }}(t)$ defined by

$\ddot{q}_{n a d}=-h_{22}^{-1} h_{21} \ddot{q}_{a d}-h_{22}^{-1} c_{21} \dot{q}_{a}-h_{22}^{-1} c_{22} \dot{q}_{n a}-h_{22}^{-1} g_{2}(q)+K_{d}^{\prime} \dot{\tilde{q}}_{n a}+K_{p}^{\prime} \tilde{q}_{n}$

thus, the closed loop equations become

$$
\begin{aligned}
& \ddot{\tilde{q}}_{a}+K_{d} \dot{\tilde{q}}_{a}+K_{p} \tilde{q}_{a}=0 \\
& \ddot{\tilde{q}}_{n a}+K_{d}^{\prime} \dot{\tilde{q}}_{n a}+K_{p}^{\prime} \tilde{q}_{n a}=h_{22}^{-1} h_{21}\left[K_{d} \dot{\tilde{q}}_{a}+K_{p} \tilde{q}_{a}\right] .
\end{aligned}
$$

The torso "reference" trajectory (17) is a function of time which satisfies the closed-loop equations and allows to analyze the stability of the origin of the closed-loop system in the sense of Lyapunov. Since this trajectory depends on the state variables it is computed online and consequently, it is not possible to predict a priori a uniform bound as $t \rightarrow \infty$. Nevertheless, for the purposes of the stability analysis, it can be shown that the system is forward complete. That is, each solution of (17), denoted $q_{\text {nad }}\left(t, t_{\circ}, q_{\text {nad }}, q_{\circ}, \dot{q}_{\circ}\right)-$ i.e., starting at time $t_{\circ}$ from the initial condition $q_{\text {nado }}$ and parameterized in the initial values of the other state variables, $q_{\circ}, \dot{q}_{\circ},-$ exists for all $t \geq t_{\circ}$ and this, for any initial conditions. The proof to this claim is omitted here due to space constraints (it is available from the authors upon request). Having said this, one may show that the following holds.

\footnotetext{
${ }^{5}$ To see this, define $\bar{H}:=\left\{\begin{array}{llll}h_{11} & h_{12} ; h_{12}^{\top} h_{22}^{-1} & 1\end{array}\right\}, \quad \tilde{H}:=$ $\left\{\begin{array}{ll}R & 0 ; h_{12}^{\top} h_{22}^{-1}\end{array} 1\right\}$ and let $h_{12, i}$ denote the $i$ th element of the column $h_{12}$. Notice that $\tilde{H}$ is obtained by subtracting the row-vector $h_{12, i}\left\{h_{12}^{\top} / h_{22}{ }^{-1} 1\right\}$ to each of the first four rows of $\bar{H}$. Hence, we have that $\operatorname{det} \tilde{H}=\operatorname{det} \bar{H}$. Furthermore, $\operatorname{det} \bar{H}=\left(\operatorname{det} H_{*}\right) / h_{22}$ and is positive since $H_{*}$ is positive definite and $h_{22}>0$. The claim follows computing $\operatorname{det} \tilde{H}$ via the leading minors corresponding to its last column, to see that $\operatorname{det} R=\operatorname{det} \tilde{H}>0$.
} 
Proposition 1 The origin of the system (15) in closed loop with (16) is globally exponentially stable (GES) around the trajectories (12), (17) for suitable diagonal positive definite matrices $K_{p}, K_{d}$, $K_{p}^{\prime}$ and $K_{d}^{\prime}$.

Roughly speaking, this result follows observing that the origin of the system (18) with the right hand side of (18b) equal to zero is GES and the right hand side of $(18 \mathrm{~b})$ is bounded (since $\left\|h_{i j}(q)\right\| \leq$ $\left.h_{M}\right)$ by a linear function of $\left\|\tilde{q}_{a} ; \dot{\tilde{q}}_{a}\right\|$.

Specifically, one may show that for sufficiently large gains the function

$$
V(\tilde{q}, \dot{\tilde{q}})=\frac{1}{2}\left(\|\dot{\tilde{q}}\|^{2}+\tilde{q}^{\top}\left[K_{P}+\varepsilon K_{D}\right] \tilde{q}\right)+\varepsilon \tilde{q}^{\top} \dot{\tilde{q}}
$$

verifies

$$
\dot{V}(\tilde{q}, \dot{\tilde{q}}) \leq-\frac{1}{2}\left(k_{D m}\|\dot{\tilde{q}}\|^{2}+\varepsilon k_{P m}\|\tilde{q}\|^{2}\right)
$$

where $\dot{V}$ is evaluated along the trajectories of (18) and $k_{D m}, k_{P m}$ are the smallest components of $K_{D}$ and $K_{P}$ respectively. The computations to obtain (20) are omitted due to space constraints.

\subsection{DS phase control}

The biped in this phase is over-actuated that is, it possesses 3 degrees of freedom and 4 controls. The dynamics of the independent coordinates (those corresponding to one leg and the torso) is given by equations of the form (14) but this time $u_{a} \in \mathbb{R}^{3}$ and $q_{n c} \in \mathbb{R}^{3}$. The control goal during this phase is to rectify the torso posture with respect to the vertical. To that end, we completely feedback linearize the system via the control law

$$
\begin{aligned}
& u_{a}:=H_{*}(q) u_{0}+C_{*}(q, \dot{q}) \dot{q}_{n c}+G_{*}(q) \\
& u_{0}=\ddot{q}_{n c d}+K_{d}^{\prime \prime}\left(\dot{q}_{n c d}-\dot{q}_{n c}\right)+K_{p}^{\prime \prime}\left(q_{n c d}-q_{n c}\right) .
\end{aligned}
$$

to obtain the exponentially stable (for positive definite diagonal gains) linearized system

$$
\ddot{\tilde{q}}_{n c}+K_{d}^{\prime \prime} \dot{\tilde{q}}_{n c}+K_{p}^{\prime \prime} \tilde{q}_{n c}=0 .
$$

We remark that during either the SS phase or the DS phase the robot is not controlled in the constrained variables, that is, in the forces directions but we assume that no sliding, bouncing or takeoff takes place during these phases.

\subsection{Stability of the complete cycle}

We have proved that the origin of the closed loop system is exponentially stable both in SS and DS phases. Asymptotic stability for almost all initial conditions (i.e., excluding initial configurations coinciding exactly with an impact phase) shall be guaranteed if the impact transition phase is such that the trajectories at the end of each impact remain in the domain where the DS-phase model is valid.

Proposition 2 Consider the system (1), (2), (11) under Assumption 1 and the standing assumption. Consider the controller (13), (16), (21), with diagonal positive definite gains. Then, the origin of the closed loop system is uniformly weakly asymptotically stable, that is, it is uniformly Lyapunov stable and all the trajectories converge to zero for almost all $t \geq 0$ and uniformly in the initial conditions.
Proof. Let $T_{i}>0$ be the time of the $i$-th impact (in general this instant is a function of $q$ but here, it is taken constant since the reference trajectories are designed so that the impacts take place at precise instants ${ }^{6}$ ). Let $T_{D S}$ and $T_{S S}$ be the duration of the double-support and single-support phases respectively (also assumed constant). Define for each $t_{\circ} \geq 0$, $I_{S S, i}:=\left\{t \in \mathbb{R}_{\geq t_{\circ}}: t \in\left[T_{i-1}^{+}+T_{D S}, T_{i}^{-}\right)\right\}, I_{I, i}:=\left[T_{i}^{-}, T_{i}^{+}\right]$, $I_{D S, i}:=\left\{t \in \mathbb{R}_{\geq t_{\circ}}: t \in\left(T_{i}^{+}, T_{i}^{+}+T_{D S}\right)\right\}$ and correspondingly, $\mathcal{I}_{S S}:=\bigcup_{i} I_{S S, i}, \mathcal{I}_{D S}:=\bigcup_{i} I_{D S, i}$ and $\mathcal{I}_{I}:=\bigcup_{i} I_{I, i}$. That is, the interval $\left[t_{\circ}, \infty\right)$ for any $t_{\circ} \geq 0$ is composed of alternated and disjoint intervals corresponding to single-support $\left(I_{S S}\right)$, impact $\left(I_{I}\right)$ and double-support $\left(I_{D S}\right)$ phases. The domains where the models for the single-support and double-support phases are valid, are respectively $\tilde{\Omega}_{S S}:=\left\{(\tilde{q}, \dot{\tilde{q}}) \in \mathbb{R}^{5} \times \mathbb{R}^{5}\right\}$ and $\tilde{\Omega}_{D S}:=\left\{(\tilde{q}, \dot{\tilde{q}}) \in \mathbb{R}^{3} \times \mathbb{R}^{3}\right.$ : $\left.\tilde{q}_{42}+q_{42 d} \neq k \pi, k \in \mathbb{N}\right\}$. With these notations, the closed loop system can be written as

$$
\begin{array}{rc}
\Sigma_{S S}: & \ddot{\tilde{q}}+K_{d} \dot{\tilde{q}}+K_{p} \tilde{q}=F(t, \tilde{q}, \dot{\tilde{q}}) \\
\Sigma_{I}: & {\left[\begin{array}{c}
q^{+} \\
\dot{q}^{+}
\end{array}\right]=\Delta(q)\left[\begin{array}{c}
q^{-} \\
\dot{q}^{-}
\end{array}\right]} \\
\Sigma_{D S}: & \ddot{\tilde{q}}+K_{d}^{\prime \prime} \dot{\tilde{q}}+K_{p}^{\prime \prime} \tilde{q}=0,
\end{array}
$$

$$
\begin{array}{r}
\forall(t, \tilde{q}, \dot{\tilde{q}}) \in \mathcal{I}_{S S} \times \tilde{\Omega}_{S}(\dot{s} \\
\forall(t, \tilde{q}, \dot{\tilde{q}}) \in \mathcal{I}_{I} \times \mathbb{R}^{2} \\
\forall(t, \tilde{q}, \dot{\tilde{q}}) \in \mathcal{I}_{D S} \times \tilde{\Omega}
\end{array}
$$

Under the standing assumption the solutions of $(\underline{23})$ are defined as right continuous functions $\varphi(\cdot)$ of bounded variation (cf. 23. 24]). They coincide with the solutions $\varphi_{S S}(\cdot)$ of $(23 \mathrm{a})$ on $\mathcal{I}_{S S, i}$ and with those of $(23 \mathrm{c})$ on the segments $\mathcal{I}_{D S, i}$.

Considering the extension of $\mathcal{I}_{S S}$ and $\mathcal{I}_{D S}$ to infinity (that is, as if the biped never finished the first step or remained grounded all the time) the respective origins of (23a) and (23c) are exponentially stable. Weak asymptotic stability of the origin of the system (23) follows if during the impact transition phases, the trajectories remain in the domain where the DS-phase model is valid. To see that this is the case, we may quantify the effect of the impact force, regarding it as a state dependent perturbation of arbitrarily short duration. Moreover, in view of (9), this force satisfies

$$
\left\|F_{\text {ext }}\right\| \leq h_{M}\left(\|\dot{\tilde{q}}\|+\left\|\dot{q}_{d}\right\|\right) \quad h_{M} \geq\|H(q)\| .
$$

Let $\beta>0$ be such that $\left\|\dot{q}_{d}(t)\right\|$ for all $t \in \mathcal{I}_{I}$. Considering the time derivative of $V(\tilde{q}, \dot{\tilde{q}})$ defined in (19) and using (20), we obtain that

$\dot{V}(\tilde{q}(t), \dot{\tilde{q}}(t)) \leq-\frac{1}{2}\left(k_{D m}\|\dot{\tilde{q}}(t)\|^{2}+\varepsilon k_{P m}\|\tilde{q}(t)\|^{2}\right)+h_{M}[\|\dot{\tilde{q}}(t)\|+\beta][\|\dot{\tilde{q}}(t)\|+\varepsilon$

for all $t \in \mathcal{I}_{S S} \cup \mathcal{I}_{I}$. Completing squares we obtain that if $k_{P m} \geq 2 h_{M}$ and $k_{D m} \geq 4 h_{M}(1+\varepsilon / 2)$ then $\dot{V}(\tilde{q}(t), \dot{\tilde{q}}(t)) \leq$ $-\frac{1}{4}\left(k_{D m}\|\dot{\tilde{q}}(t)\|^{2}+\varepsilon k_{P m}\|\tilde{q}(t)\|^{2}\right)+\beta h_{M}[\|\dot{\tilde{q}}(t)\|+\varepsilon\|\tilde{q}(t)\|]$ for all $t \in \mathcal{I}_{S S} \cup \mathcal{I}_{I}$. On the other hand, letting $\left(\tilde{q}^{*}, \dot{\tilde{q}}^{*}\right)$ be any vectors such that $\left\|\tilde{q}^{*}\right\|=\frac{8 \beta h_{M}}{K_{P m}},\left\|\dot{\tilde{q}}^{*}\right\|=\frac{8 \beta h_{M}}{K_{D m}}$ and $c:=V\left(\tilde{q}^{*}, \dot{\tilde{q}}^{*}\right)$ we obtain that $\dot{V}(\tilde{q}(t), \dot{\tilde{q}}(t)) \leq-\frac{1}{8}\left(k_{D m}\|\dot{\tilde{q}}(t)\|^{2}+\varepsilon k_{P m}\|\tilde{q}(t)\|^{2}\right)$ on the set $\{(t, \tilde{q}, \dot{\tilde{q}}): V(\tilde{q}(t), \dot{\tilde{q}}(t)) \geq c\}$. It follows that $\dot{V}(\tilde{q}(t), \dot{\tilde{q}}(t))$ and, consequently $\|\tilde{q}(t) ; \dot{\tilde{q}}(t)\|$, tend to a closed domain which can be made small by enlarging the control gains. In words, the perturbation due to the impact may be compensated for to ensure that each DS phase starts off with the right initial conditions. Exponential stability for almost all $t$ follows since the origin of (23c) is also exponentially stable.

\footnotetext{
${ }^{6}$ If non constant $T_{i}$ 's are to be considered the analysis also holds under suitable assumptions which roughly, are satisfied if the solutions cross the section $S(q, \dot{q})=0$ which defines the state-dependent impacts configurations. This assumption holds if the non-sliding and contact constraints are satisfied. See e.g. [24, 6].
} 
Remark 1 The argument above is based on the assumption that $\left\|\dot{q}_{d}\left(T_{i}\right)\right\| \leq \beta_{i}$ at the moment of impact. Even though it is not possible to quantify in general the number $\beta_{i}$ due to the dependence of $\dot{q}_{\text {nad }}$ on the system's trajectories and on the impact, the latter is arbitrarily short in time hence, one may expect that the bound on $q_{\text {nad }}$ on the interval of interest is also relatively small. Notice that it is not needed that $\dot{q}_{\text {nad }}(t)$ be bounded by $\beta$ for all time for each set of initial conditions but rather that it be bounded on compact intervals. This is ensured since the solutions of (23a) exist and are unique for all $t$.

\section{Simulation Results}

In the last page we present some simulation results obtained for a 7 degrees-of-freedom biped. We have used the simulator created for the prototype of the project "Legged Robots" (cf. http://www-lag.ensieg.inpg.fr/PRC-Bipedes/) sponsored by the CNRS, France.

We have fixed the duration of a complete cycle (hence the gait's period) to $1.4286 \mathrm{sec}$. The SS phase constitutes $70 \%$ i.e., it lasts $1 \mathrm{~s}$ while the DS phase lasts $30 \%$ of the cycle i.e., 0.4286 s. The impact is considered to be instantaneous hence, this has been carefully taken into account by resetting the simulation with different initial conditions at each impact. The parameter $\alpha=20^{\circ}$ (cf. reference trajectories).

We show for one leg and the torso the articular positions in Fig. 1. The intervals over which the positions are constant correspond to DS phases. One may also appreciate that for the unactuated coordinate i.e the torso's, the deviation for the first step is about $8^{\circ}$ but this error is asymptotically corrected during the succeeding DS phases. In particular, notice that the coordinate of the torso remains bounded as expected since the system is forward complete. It has been observed in simulations that the bound on $q_{n a}$ depends on different parameters such as the length and size of a step but a general rule cannot be established formally. In Fig. 2 we show the corresponding velocities of the same leg and the torso. Fig. 3 depicts two of the input torques which remain within the maximal acceptable boundaries for our prototype (150N). Finally, we show the reaction tangential and normal forces for both legs in Fig. 4. One may appreciate the switching of phases during which the normal components are zero, these corresponds to the phases of SS when one foot is in the air.

\section{Conclusion}

We have addressed the problem of trajectory control of a planar 7 degrees-of-freedom under-actuated bipedal robot. Our approach consists on controlling the system independently in the phases of SS and DS while ensuring a certain degree of robustness during the impact phase. Our work is inspired by previous results for mechanical systems under holonomic and unilateral constraints. We have shown the performance of our controllers on a simulator of a real biped robot. We expect that experimental results and more precise theoretical arguments will be available soon. Present research is carried out on the domain of generation (on line) of optimal trajectories compatible with all the constraints involved in the system's model.

\section{REFERENCES}

[1] M. H. Raibert, "Legged robots," Commmun. ACM, vol. 29, pp. $499-514,1986$.
[2] Y. Aoustin and A. Formal'sky, "Stability of a cyclic biped gait and hastening of the convergence to it," in Int. Conf. on Climbing and Walking Robots, (Karlsruhe, Germany), pp. 779-788, 2001.

[3] A. Chevallereau, "Parametrized control for an underactuated biped robots," in Proc. 15th. IFAC World Congress, (Barcelona, Spain), 2001.

[4] A. Formal'sky, C. Chevallereau, and B. Perrin, "On ballistic walking locomotion of a quadruped," Int. J. Robotics Res., vol. 19, no. 8, pp. 743-761, 2000.

[5] R.Katoh and M. Mori, "Control method of biped locomotion giving asymptotic stability of trajectory," Automatica, vol. 20, no. 4, pp. 405-414, 1984.

[6] J. W. Grizzle, G. Abba, and F. Plestan, "Asymptotically stable walking for biped robots: analysis via systems with impulse effects," IEEE Trans. on Automat. Contr., vol. 46, pp. 51-64, January 2000.

[7] F. Plestan, J. W. Grizzle, E. Westervelt, and G. Abba, "Stable walking of a 7-dof biped robot," IEEE Trans. on Robotics Automat., vol. 19, no. 4, 2003.

[8] Y. Aoustin and A. Formal'sky, "Conception d'une loi de commande d'un robot bipde dont les articulations motorises sont fonction d'une variable non commande," in CIFA 2000, pp. 840-845, 2000.

[9] E. Westervelt, J. Grizzle, and D. Koditschek, "Hybrid zero dynamics of planar biped walkers," IEEE Trans. on Automat. Contr., vol. 48, pp. 42-56, 2003.

[10] C. Chevallereau and P. Sardain, "Design and actuation optimization of a 4 axes biped robot for walking and running," in Proc. IEEE Conf. Robotics Automat., (San Francisco, CA-USA), pp. 3365-3370, 2000 .

[11] A. Loría and E. Panteley, "Force/tracking control of constrained manipulators without velocity measurements," IEEE Trans. on Automat. Contr., vol. 44, no. 7, pp. 1407-1412, 1999.

[12] M. Spong, "The control of underactuated mechanical systems," in 1st Int. Conf. on Mechatronics, (Mexico City), 1994.

[13] M. Spong and M. Vidyasagar, Robot Dynamics and Control. New York: John Wiley \& Sons, 1989.

[14] L. Sciavicco and B. Siciliano, Modeling and control of robot manipulators. New York: McGraw Hill, 1996.

[15] B. Brogliato, S. Niculescu, and P. Orhant, "On the control of finite-dimensional mechanical systems with unilateral constraints," IEEE Trans. on Automat. Contr., vol. 42, no. 2, pp. 200-216, 1997.

[16] E. Panteley and A. Stotsky, "Adaptive trajectory/force control scheme for constrained robot manipulators," Int. J. Adapt. Contr. Sign. Process., vol. 7, no. 6, pp. 489-496, 1993.

[17] E. Panteley and A. Stotsky, "Asymptotic stability of constrained robot motion observer based control schemes," in Proc. 2nd. European Contr. Conf., (Groningen, The Netherlands), pp. 1255-1260, 1993.

[18] I. Sandberg, "Global implicit function theorems," IEEE Trans. Circuits and Systems, vol. 28, pp. 145-149, 1981.

[19] F. R. Gantmacher, The theory of matrices, vols. I and II. New York: Chelsea publishing Co., 1960.

[20] H. McClamroch and D. Wang, "Feedback stabilization and tracking of constrained robots," IEEE Trans. on Automat. Contr., vol. 33, pp. 419-426, 1988.

[21] A. Chemori, Commande des Robots bipdes. LAG - INPG: Rapport de DEA, 2001.

[22] Y. Hurmuzlu and D. Marghitu, "Rigid body collisions of planar kinematic chains with multiple contact points," Int. J. Rob. Research, vol. 13, no. 1, pp. 82-92, 1994.

[23] B. Brogliato, Nonsmooth impact mechanics. Models, dynamics and control. No. 220 in LNCIS, Springer Verlag, 1996.

[24] T. Yang, Impulsive control theory. No. 272 in LNCIS, SpringerVerlag, 2001.

[25] H. Khalil, Nonlinear systems. New York: Macmillan Publishing Co., 2nd ed., 1996.

[26] J. Hale, Ordinary Differential equations. Interscience, New York: John Wiley, 1969. 

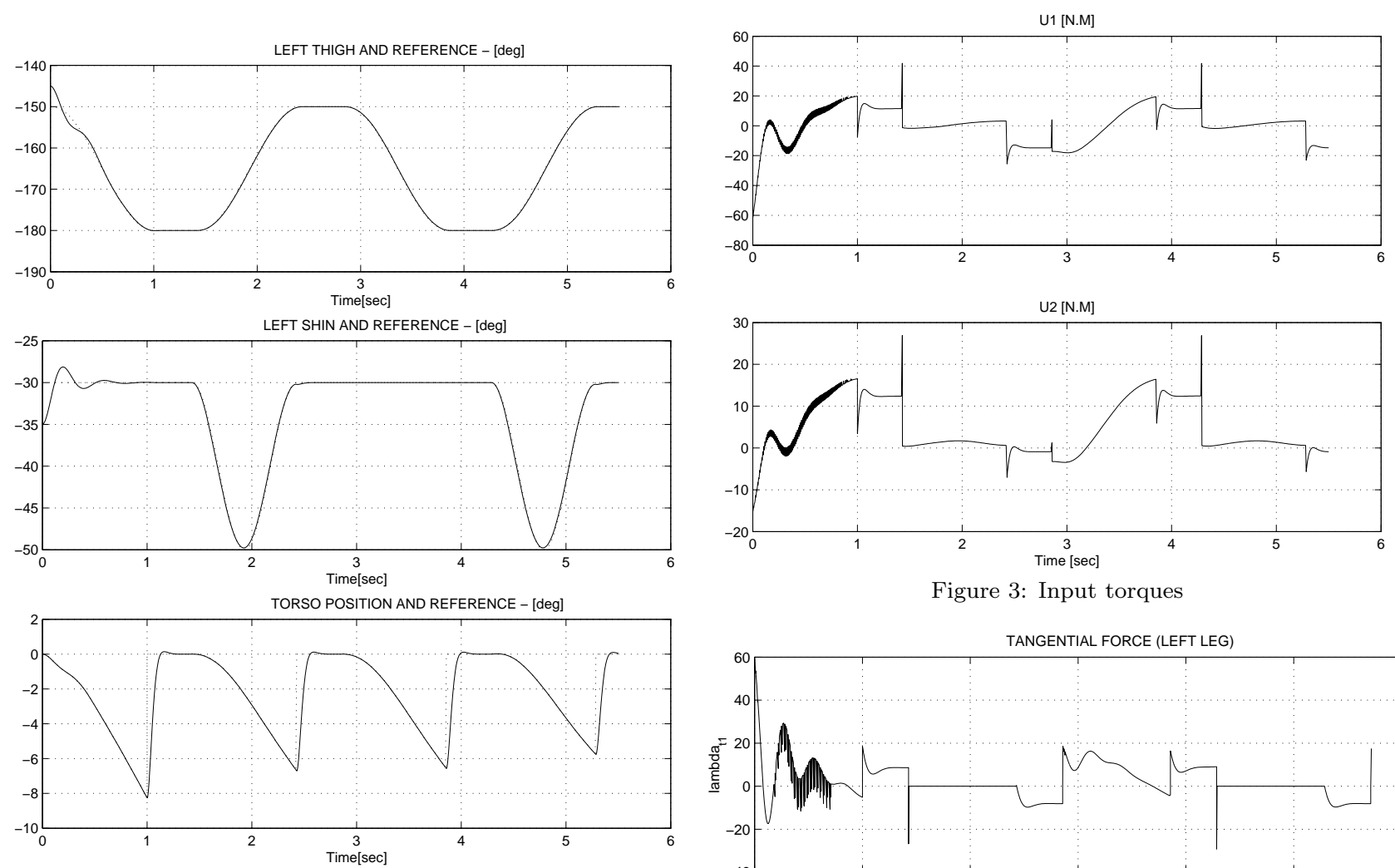

Figure 3: Input torques

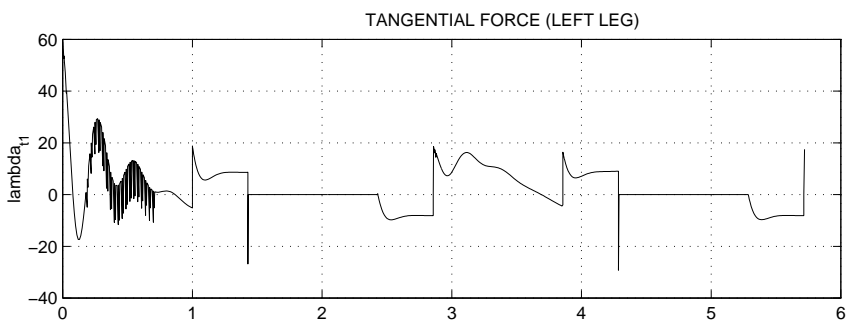

Figure 1: Articular positions of left leg \& torso
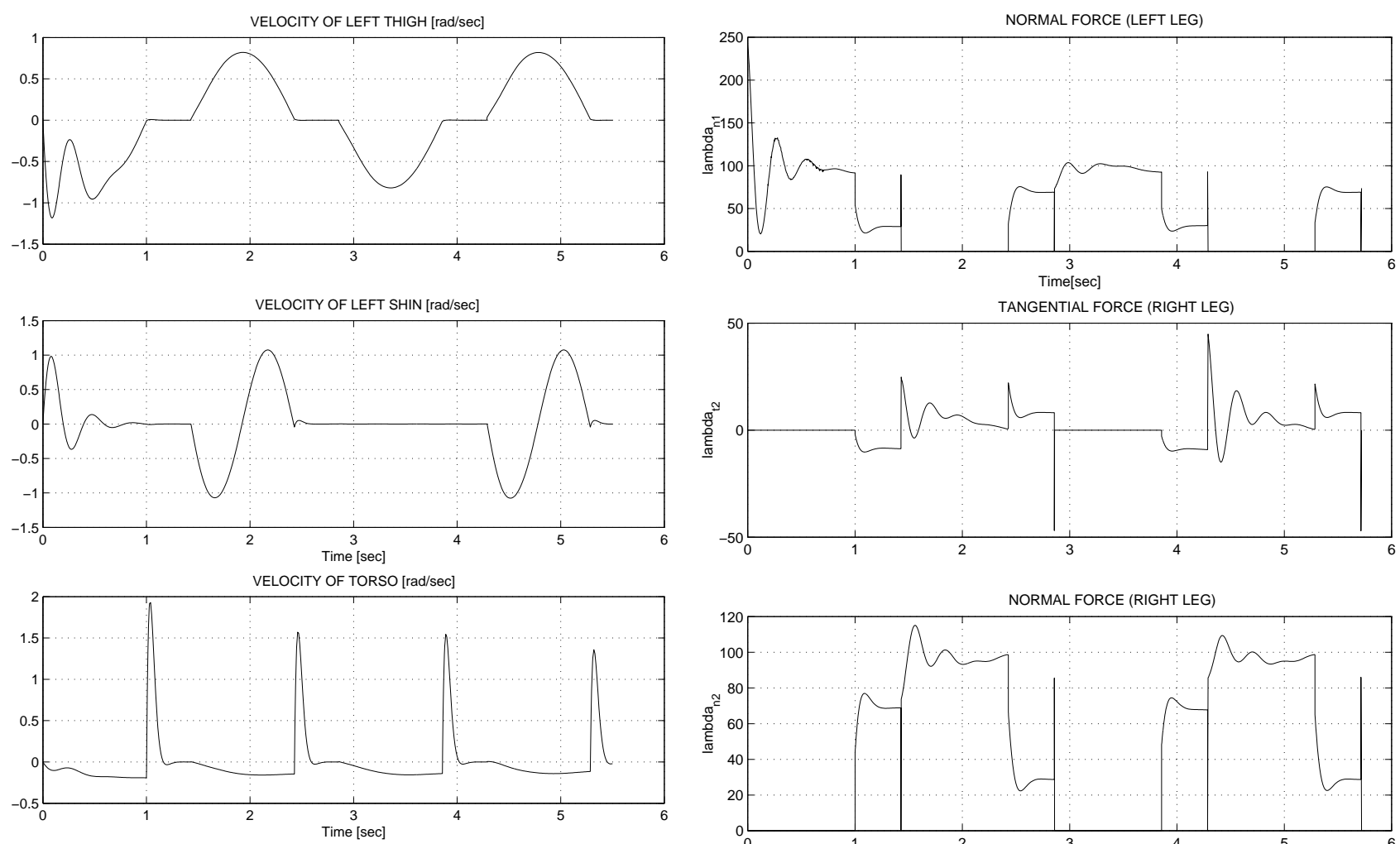

Figure 2: Articular velocities of left leg \& torso

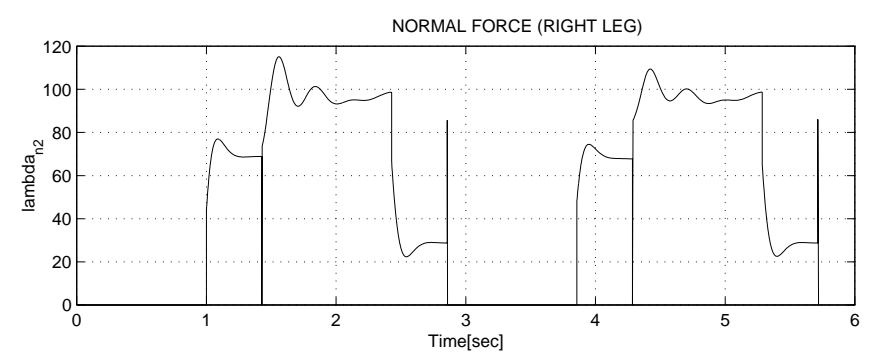

Figure 3: Reaction forces 